COMMUNICATIONS IN

ANALYSIS AND GEOMETRY

Volume 14, Number 4, 625-632, 2006

\title{
A remark on lower bound of Milnor number and characterization of homogeneous hypersurface singularities
}

\author{
Ke-Pao Lin, Xi Wu, Stephen S.-T. Yau and Hing-Sun Luk
}

\begin{abstract}
Let $f:\left(\mathcal{C}^{n+1}, 0\right) \rightarrow(\mathcal{C}, 0)$ be a holomorphic germ defining an isolated hypersurface singularity $\mathrm{V}$ at the origin. Let $\mu$ and $\nu$ and $p_{g}$ be the Milnor number, multiplicity and geometric genus of $(V, 0)$, respectively. We conjecture that $\mu \geq(\nu-1)^{n+1}$ and the equality holds if and only if $f$ is a semi-homogeneous function. We prove that this inequality holds for $n=1$, and also for $n=2$ or 3 with additional assumption that $f$ is a quasihomogeneous function. For $n=1$, if $V$ has at most two irreducible branches at the origin, or if $f$ is a quasi-homogeneous function, then $\mu=(\nu-1)^{2}$ if and only if $f$ is a homogeneous polynomial. For $n=2$, if $f$ is a quasihomogeneous function, then $\mu=(\nu-1)^{3}$ iff $6 p_{g}=\nu(\nu-1)(\nu-2)$ iff $f$ is a homogeneous polynomial after biholomorphic change of variables. For $n=3$, if $f$ is a quasi-homogeneous function, then $\mu=(\nu-1)^{4}$ iff $24 p_{g}=\nu(\nu-1)(\nu-2)(\nu-3)$ iff $f$ is a homogeneous polynomial after biholomorphic change of variables.
\end{abstract}

\section{Introduction}

Since the fundamental work of Milnor [1] on isolated hypersurface singularities, a principal tool in the study of topology of isolated singularities has been the Milnor fibration of the singularity. Let $f:\left(\mathbb{C}^{n+1}, 0\right) \rightarrow(\mathbb{C}, 0)$ be a holomorphic germ defining an isolated hypersurface singularity so that for local coordinates $\left(z_{0}, z_{1}, \ldots, z_{n}\right)$ the partials $\partial f / \partial z_{i}$ do not simultaneously vanish in a punctured neighborhood of zero. Milnor associates to $f$ a fibration, defined for $\epsilon>0$ and $\delta>0$ sufficiently small,

$$
f^{-1}\left(D_{\delta}^{*}\right) \cap B_{\epsilon} \longrightarrow D_{\delta}^{*}
$$

where $B_{\epsilon}$ denotes a ball of radius $\epsilon$ about 0 in $\mathbb{C}^{n+1}, D_{\delta}$ denotes the disk with radius $\delta$ about 0 in $\mathbb{C}$ and $D_{\delta}^{*}=D_{\delta} \backslash\{0\}$. This fibration has 
fiber $V_{t}=f^{-1}(t) \cap B_{\epsilon}$, which is the Milnor fiber of the singularity $V_{0}=$ $\left\{z \in \mathbb{C}^{n+1}: f(z)=0\right\}$. Then, Milnor proves:

Theorem 1.1 [1]. If $f$ has an isolated singularity at 0 , then for $\epsilon>0$ and $\delta>|t|>0$ sufficiently small:

(1) The Milnor fibration is a smooth fiber bundle, with the diffeomorphism type of the fiber $V_{t}$ independent of $\epsilon$ and $t$.

(2) The Milnor fiber $V_{t}$ is homotopy equivalent to the bouquet of spheres of real dimension $n$, in particular, the Milnor fiber is $(n-1)$-connected.

(3) The number of such spheres (which are the "vanishing cycles") is called the Milnor number and can be computed by the formula

$$
\mu(f)=\mu\left(V_{0}\right)=\frac{\operatorname{dim} \mathbb{C}\left\{z_{0}, \ldots, z_{n}\right\}}{\left(\partial f / \partial z_{0}, \partial f / \partial z_{1}, \ldots, \partial f / \partial z_{n}\right)} .
$$

Milnor number is an important tool to study the topology of singularity. For example, Lê and Ramanujam [2] have proved the following important theorem.

Theorem $1.2[2,3]$. Let $f_{t}:\left(\mathbb{C}^{n+1}, 0\right) \rightarrow(\mathbb{C}, 0)$ be a family of germs of holomorphic maps, smoothly depending on the parameter $t \in \mathbb{R}^{p}$. Suppose that for any $t$, the Milnor number $\mu_{t}$ of the germs $f_{t}$ is finite and $\mu_{t}$ does not depend on $t$. Suppose also that $n \neq 2$. Then all the germs $f_{t}$ are topologically equivalent.

Let $G^{(i)}$ be the Grassmannian of $i$-planes in $\mathbb{C}^{n+1}$. Teissier [4] proves that there exists a Zariski-open dense $U^{(i)} \subseteq G^{(i)}$ such that $\mu(f / H)=\mu^{(i)}(f)$ for all $H \in U^{(i)}$. Notice that $\mu^{(n+1)}(f)=\mu(f), \mu^{(1)}(f)=\nu-1$, where $\nu$ is the multiplicity of $f$ at 0 , and $\mu^{(0}(f)=1$. Set $\mu^{*}(f)=\left(\mu^{(n+1)}(f), \ldots, \mu^{(1)}(f)\right.$, $\left.\mu^{(0)}(f)\right)$.

Let $\lambda: \mathcal{V} \rightarrow T$ be the germ of a flat deformation of the two-dimension isolated hypersurface singularity $(V, 0)$. We take $T$ to be reduced. In [5], Teissier introduced, for all dimensions, various notions of simultaneous resolution of $\lambda$. Namely, let $V_{t}$ denote $\lambda^{-1}(t)$, the fiber above $t$ in $T$.

Definition 1.3. The map germ $\prod: \mathcal{M} \rightarrow \mathcal{V}$ is a very weak simultaneous resolution of $\lambda$ if for all sufficiently small representatives of $\lambda$, the germ $\Pi$ has a representative, also denoted $\prod$, such that

(i) $\prod$ is a proper modification map; 
(ii) $\lambda \circ \prod: \mathcal{M} \rightarrow T$ is a flat map;

(iii) $\prod_{t}: M_{t} \rightarrow V_{t}$ is a resolution of $V_{t}$ for all $t$.

Definition 1.4. With the notation in Definition 1.3, consider $V$ to have dimension 2. Let $\mathcal{A}$ denote the exceptional set in $\mathcal{M}$. $\prod$ is a weak simultaneous resolution if additionally the map induced by restriction $\overline{\lambda \circ \prod}: \mathcal{A} \rightarrow T$ is simple, i.e., a locally trivial deformation.

Definition 1.5. With the notations in Definition 1.3 and Definition 1.4, let $\mathcal{L}$ denote the singular locus of $\mathcal{V}$. Consider $\prod^{-1}(\mathcal{L})$ as nonreduced analytic space (with $\mathcal{A}$ as its underlying reduced space). $\prod$ is a strong simultaneous resolution if in addition to (i), (ii) and (iii) in Definition 1.3, the map induced

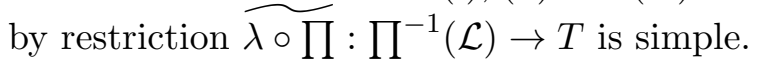

The following beautiful theorem was proved by Laufer [6].

Theorem 1.6 [6]. Let $\lambda: \mathcal{V} \rightarrow T$, with $T$ reduced, be a (flat) family of isolated hypersurface two-dimensional singularities. Suppose that $\mu^{*}\left(V_{t}\right)$ is constant as a function of $t$. Then $\lambda$ has a strong simultaneous resolution.

It is clear that Milnor number is an important numerical invariant which measures the complexity of the singularity. Therefore, it is desirable to give a lower bound of the Milnor number.

Conjecture 1.7. Let $f:\left(\mathbb{C}^{n+1}, 0\right) \rightarrow(\mathbb{C}, 0)$ be a holomorphic germ defining an isolated hypersurface singularity $V=\left\{z \in \mathbb{C}^{n+1}: f(z)=0\right\}$ at the origin. Let $\mu$ and $\nu$ be the Milnor number and multiplicity of $(V, 0)$, respectively. Then

$$
\mu \geq(\nu-1)^{n+1}
$$

and the equality in (1.1) holds if and only if $f$ is a semi-homogeneous function (i.e., $f=f_{\nu}+g$, where $f_{\nu}$ is a nondegenerate homogeneous polynomial of degree $\nu$ and $g$ consists of terms of degree at least $\nu+1)$. Suppose that $f$ is a quasihomogeneous function, i.e., $f \in\left(\partial f / \partial z_{0}, \ldots, \partial f / \partial z_{n}\right)$. Then the equality in (1.1) holds if and only if $f$ is a homogeneous polynomial (after a biholomorphic change of coordinates).

The purpose of this paper is to prove the following theorems. 
Theorem 1.8. Let $f:\left(\mathbb{C}^{2}, 0\right) \rightarrow(\mathbb{C}, 0)$ be a holomorphic germ defining an isolated plane curve singularity $V=\left\{z \in \mathbb{C}^{2}: f(z)=0\right\}$ at the origin. Let $\mu$ and $\nu$ be the Milnor number and multiplicity of $(V, 0)$, respectively. Then

$$
\mu \geq(\nu-1)^{2} .
$$

Furthermore, if $V$ has at most two irreducible branches at the origin, or if $f$ is a quasi-homogeneous function, then the equality in (1.2) holds if and only if $f$ is a homogeneous polynomial (after a biholomorphic change of coordinates).

Theorem 1.9. Let $f:\left(\mathbb{C}^{n+1}, 0\right) \rightarrow(\mathbb{C}, 0)$ be a holomorphic germ defining an isolated hypersurface singularity $V=\left\{z \in \mathbb{C}^{n+1}: f(z)=0\right\}$ at the origin. Let $\mu, \nu$ and $\tau=\operatorname{dim} \mathbb{C}\left\{z_{0}, \ldots, z_{n}\right\} /\left(f, \partial f / \partial z_{0}, \ldots, \partial f / \partial z_{n}\right)$ be the Milnor number, multiplicity and Tjurina number of $(V, 0)$, respectively. Suppose $\mu=\tau$ and $n$ is either 2 or 3. Then

$$
\mu \geq(\nu-1)^{n+1}
$$

and the equality in (1.3) holds if and only if $f$ is a homogeneous polynomial (after a biholomorphic change of coordinates).

\section{Plane curve singularities}

The purpose of this section is to prove Theorem 1.8 in the previous section for plane curve singularities. Let $(V, 0) \subseteq\left(\mathbb{C}^{2}, 0\right)$ be a singularity with $r$ irreducible components. It is well known that (see p. 574 of [7])

$$
\mu=\sum \nu_{i}\left(\nu_{i}-1\right)-r+1,
$$

where $\nu_{i}$ runs through the multiplicities of the strict preimages of $V$ at all infinitely near points of $0 \in V$. In particular,

$$
\mu \geq \nu(\nu-1)-r+1=(\nu-1)^{2}+\nu-r .
$$

Since multiplicity of the singularity is bigger than or equal to the number of irreducible components of the singularity (i.e., $\nu-r \geq 0$ ), (2.2) implies

$$
\mu \geq(\nu-1)^{2},
$$

and the equality in (2.3) occurs if and only if $(V, 0)$ can be resolved by one quadratic transformation and $\nu=r$. Observe that $\nu=r$ means that each 
irreducible component of $(V, 0)$ is smooth. These irreducible components intersect transversely because $(V, 0)$ can be resolved by one quadratic transformation. Therefore, by the hypothesis of Theorem 1.8, equality of (2.3) occurs if and only if $f$ is a homogeneous polynomial after a biholomorphic change of coordinates.

\section{Surface singularities}

In this section, we shall prove Theorem 1.9 for surface singularities. Since $\mu(f)=\tau(f)$, by a theorem of Saito [8], $f$ is a weighted homogeneous polynomial after a biholomorphic change of coordinates. Xu-Yau's theorem [9] asserts that

$$
\mu \geq 6 p_{g}+\nu-1
$$

and the equality in (3.1) holds if and only if $f$ is a homogeneous polynomial. Observe that (3.1) can be rewritten as

$$
\mu \geq 6 p_{g}-\nu(\nu-1)(\nu-2)+(\nu-1)^{3} .
$$

We claim that $6 p_{g} \geq \nu(\nu-1)(\nu-2)$. To see this, let us recall a beautiful theorem of Merle and Teissier [10]. Let $f\left(z_{0}, \ldots, z_{n}\right)$ be a germ of analytic functions at the origin such that $f(0)=0$. Suppose $f$ has an isolated critical point at the origin. $f$ can be developed in a convergent Taylor series $f\left(z_{0}, \ldots, z_{n}\right)=\sum a_{\lambda} z^{\lambda}$, where $z^{\lambda}=z_{0}^{\lambda_{0}} \cdots z_{n}^{\lambda_{n}}$. Recall that Newton boundary $\Gamma(f)$ is the union of the compact faces of $\Gamma_{+}(f)$, where $\Gamma_{+}(f)$ is the convex hull of the union of the subsets $\left\{\lambda+\left(\mathbb{R}_{+}\right)^{n+1}\right\}$ for $\lambda$ such that $a_{\lambda} \neq 0$. Finally, let $\Gamma_{-}(f)$, the Newton polyhedron of $f$, be the cone over $\Gamma(f)$ with cone point at 0 . For any closed face $\Delta$ of $\Gamma(f)$, we associate the polynomial $f_{\Delta}(x)=\sum_{\lambda \in \Delta} a_{\lambda} x^{\lambda}$. We say that $f$ is nondegenerate if $f_{\Delta}$ has no critical point in $\left(\mathbb{C}^{*}\right)^{n+1}$ for any $\Delta \in \Gamma(f)$, where $C^{*}=\mathbb{C}-\{0\}$. We

say that a point $p$ of the integral lattice $\mathbb{Z}^{n+1}$ in $\mathbb{R}^{n+1}$ is positive if all the coordinates of $p$ are positive.

Theorem 3.1 [10]. Let $(V, 0)$ be a isolated hypersurface singularity defined by a nondegenerate holomorphic function $f:\left(\mathbb{C}^{n+1}, 0\right) \rightarrow(\mathbb{C}, 0)$. Then the geometric genus $p_{g}=\#\left\{p \in \mathbb{Z}_{+}^{n+1} \cap \Gamma_{-}(f): p\right.$ is positive $\}$.

Now the claim $6 p_{g} \geq \nu(\nu-1)(\nu-2)$ follows from Merle-Teissier theorem because $\Gamma_{-}(f)$ contains the tetrahedron with vertices $(0,0,0),(\nu, 0,0)$, $(0, \nu, 0)$ and $(0,0, \nu)$ which contains $\frac{1}{6} \nu(\nu-1)(\nu-2)$ positive integral points. 
It is also clear that $6 p_{g}=\nu(\nu-1)(\nu-2)$ if and only if $\Gamma_{-}(f)$ coincide with this tetrahedron, i.e., $f$ is a homogeneous polynomial. Therefore, (3.2) implies

$$
\mu \geq(\nu-1)^{3}
$$

and equality in (3.3) holds if and only if $6 p_{g}=\nu(\nu-1)(\nu-2)$, which holds if and only if $f$ is a homogeneous polynomial.

Corollary 3.2. Let $f:\left(\mathbb{C}^{3}, 0\right) \rightarrow(\mathbb{C}, 0)$ be a holomorphic germ defining on isolated hypersurface singularity $V=\left\{z \in \mathbb{C}^{3}: f(z)=0\right\}$ at the origin. Let $\mu, \nu$ and $\tau$ be the Milnor number, multiplicity and Tjurina number of $(V, 0)$, respectively. Suppose $\mu=\tau$. Then

$$
\begin{aligned}
\mu & \geq(\nu-1)^{3}, \\
6 p_{g} & \geq \nu(\nu-1)(\nu-2),
\end{aligned}
$$

and equality in (3.4) holds if and only if equality in (3.5) holds, which holds if and only if $f$ is a homogeneous polynomial after biholomorphic change of coordinates.

\section{Three-dimensional singularities}

The same method in the previous section can be used to prove Theorem 1.9 for three-dimensional singularities. Instead of using $\mathrm{Xu}$-Yau's theorem, we use Lin-Yau's theorem $[11,12]$ which asserts that

$$
\begin{aligned}
\mu & \geq 4 ! p_{g}+2 \nu^{3}-5 \nu^{2}+2 \nu+1 \\
& =4 ! p_{g}+(\nu-1)^{4}-\nu(\nu-1)(\nu-2)(\nu-3) .
\end{aligned}
$$

Corollary 4.1. Let $f:\left(\mathbb{C}^{4}, 0\right) \rightarrow(\mathbb{C}, 0)$ be a holomorphic germ defining an isolated hypersurface singularity $V=\left\{z \in \mathbb{C}^{4}: f(z)=0\right\}$ at the origin. Let $\mu, \nu$ and $\tau$ be the Milnor number, multiplicity and Tjurina number of $(V, 0)$, respectively. Suppose $\mu=\tau$. Then

$$
\begin{aligned}
\mu & \geq(\nu-1)^{4}, \\
24 p_{g} & \geq \nu(\nu-1)(\nu-2)(\nu-3),
\end{aligned}
$$

and equality in (4.2) holds if and only if equality in (4.3) holds, which holds if and only if $f$ is a homogeneous polynomial after biholomorphic change of coordinates. 


\section{Acknowledgment}

Stephen S.-T. Yau is supported in part by NSA, Zi-Jiang Professor of East China Normal University, and Hong Kong RGC. Hing-Sun Luk is supported in part by Hong Kong RGC.

\section{References}

[1] J. Milnor, Singular Points of Complex Hypersurfaces, Ann. of Math. Studies 61, Princeton University Press, Princeton, NJ, 1968.

[2] D. Lê and C. Ramanujan, The invariance of Milnor's number implies the invariance of the topological type, Amer. J. Math., 98 (1976), $67-78$.

[3] I.G. Timourian, The invariance of Milnor's number implies topological triviality, Amer. J. Math., 99 (1977), 437-446.

[4] B. Teissier, Introduction to equisingularity problem, Proceedings of the Symposia in Pure Mathematics, AMS, 29 (1975), 593-642.

[5] B. Teissier, Resolution simultanée I, II, Seminaire sur les Singularities des Surfaces, ed. Demazure et al., Lect. Notes Math., 777, SpringerVerlag, Berlin, 1980.

[6] H. Laufer, Strong simultaneous resolution for surface singularities, Advanced Studies in Pure Mathematics 8, Complex Analytic Singularities, 1986, 207-214.

[7] E. Brieskorn and H. Knörrer, Plane Algebraic Curve, Birkhäuser Verlag, 1986.

[8] K. Saito, Quasihomogene isolierte singularitäten von Hyperflachen, Invent. Math., 14 (1971), 123-142.

[9] Y.-J. Xu and S.S.-T. Yau, Durfee conjecture and coordinate free characterization of homogeneous singularities, J. Diff. Geom., 37 (1993), $375-396$.

[10] M. Merle and B. Teissier, Conditions d'adjonction d'aprěs Du Val, Seminaire Sur les Singularities des Surfaces, ed. Demazure et al., in Lect. Notes Math., 777, Springer-Verlag, Berlin, 1980, 229-245. 
[11] K.-P. Lin and S.S.-T. Yau, Classification of affine varieties being cones over nonsingular projective varieties: hypersurface case, communication in analysis and geometry, 12(5) (2004), 1201-1219.

[12] K.-P. Lin, Z.-H. Tu and S.S.-T. Yau, Characterization of isolated homogeneous hypersurface singularities in $\mathbb{C}^{4}$. Science in China Series A: Mathematics, 49(11) (2006), 1576-1592.

Department of Information MANAGEMENT Chang Gung Institute of Technology

261 Wen HWa 1 RoAD

KWEI-SHEN, TAO-YUAN

TAIWAN

RePublic of China

E-mail address: kplin@mail.cgit.edu.tw

Department of Mathematics, Statistics And Computer Science (M/C 249)

University of IlLINOIS AT ChicAGo

851 South Morgan Street

Chicago, IL 60607-7045

USA

E-mail address: yau@uic.edu

DePARTMENT OF MATHEMATiCS

Chinese University of Hong Kong

Shatin, NT

HONG KONG

E-mail address: hsluk@math.cuhk.edu.hk

Received July 11, 2006 